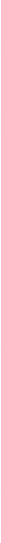

\title{
Propuesta de evaluación cultural de Bienes Muebles de Patrimonio religioso
}

\author{
Maite Barrio Olano, Ion Berasain Salvarredi, Jesús Muñiz Petralanda, Ignacio Migueliz Valcarlos, \\ María Pía Timón Tiemblo, Itziar de Azua Brea
}

Resumen: El valor cultural de un objeto es un concepto complejo y amplio, fruto de una multiplicidad de factores y significados. Su evaluación, indispensable para la buena gestión de su protección y conservación, se convierte en tarea difícil y delicada al enlazarse este conjunto de variables. El artículo presenta un formulario de valoración diseñado a partir de la identificación de una serie de valores y subvalores propios del bien cultural y de su baremación verbal y numérica. Esta herramienta ha sido utilizada en un proyecto de revisión de inventario de bienes muebles religiosos de una pequeña población guipuzcoana, con el fin de jerarquizar dichos bienes con cierto grado de objetividad y facilitar de esta manera un planteamiento posterior de niveles de protección.

Palabras clave: evaluación cultural, valor cultural, criterio, bien cultural mueble, protección, colección, patrimonio cultural

\section{Cultural assessment proposal for religious heritage objects}

Abstract: The cultural value of any object is an extremely complex, broad concept, the compendium of multiple factors and interpretations. The evaluation of any object - necessary for its proper protection and conservation - becomes even more complicated and delicate when this particular set of variables comes into play. This article contemplates an evaluation form designed according to the identification of a series of values and sub-values peculiar to cultural assets and their verbal and numerical tabulation. This tool has been used for a revision of inventory of movable religious heritage in a small population in Gipuzkoa, in order to prioritize this heritage with some degree of objectivity and thus facilitate this subsequent determination of the necessary levels of protection.

Key words: cultural assessment, cultural value, judgment, movable cultural property, protection, collection, cultural heritage

\section{Proposta de avaliação cultural de bens moveis do património religioso}

Resumo: O valor cultural de um objecto é um conceito complexo e amplo, fruto de uma multiplicidade de factores e significados. A sua avaliação, indispensável a uma boa gestão da protecção e da conservação do objecto, torna-se numa tarefa difícil e delicada ao relacionar-se com este tipo de variáveis. $\mathrm{O}$ artigo apresenta uma grelha de avaliação desenhada a partir da identificação de uma série de valores e de sub-valores inerentes ao bem cultural e da sua classificação verbal e numérica. Esta ferramenta foi empregue num projeto de revisão de inventário de bens religiosos móveis de uma pequena população de Guipúscoa, com o objectivo de hierarquizar esses bens com um certo grau de objectividade, tendo em vista uma definição posterior de níveis de protecção.

Palavras-chave: Avaliação cultural, valor cultural, criterio, bem cultural móvel, protecção, colecção, patrimonio cultural 


\section{Evaluación cultural}

El patrimonio cultural de un país o de una región está constituido por todos aquellos bienes materiales e inmateriales, naturales, inmuebles o muebles, portadores de valor cultural. Ahora bien, ¿Cómo se define el valor cultural? ¿Cómo se decide que un objeto lo posee o lo representa? El concepto es complejo y muy amplio y a menudo resulta impreciso, fruto de una multiplicidad de factores que lo componen y de una acumulación de significados [figura 1].

Quizá por ello, y a pesar de la importante reflexión teórica desarrollada en las últimas décadas ${ }^{1}$, la evaluación cultural de un bien $y$, en concreto, de un bien mueble, no es una práctica frecuente ${ }^{2}$. A la hora de llevar a cabo inventarios o sistemas de documentación es un campo que raramente se contempla y, en el mejor de los casos, suele estar remplazado por el valor económico, entendido como precio-mercado, concepto este mucho más restringido y aleatorio.

La valoración de un objeto es una tarea delicada puesto que en cualquier bien cultural se conjugan una serie de valores materiales e inmateriales que lo conforman y justifican su inserción en la categoría de patrimonio. Críticos, técnicos e historiadores, realizan frecuentemente valoraciones de los bienes que dependen únicamente de su rigor profesional, pudiendo caer fácilmente en apreciaciones subjetivas y parciales.

Sin embargo, la jerarquización del valor de un conjunto de bienes muebles, a pesar de su dificultad, resulta

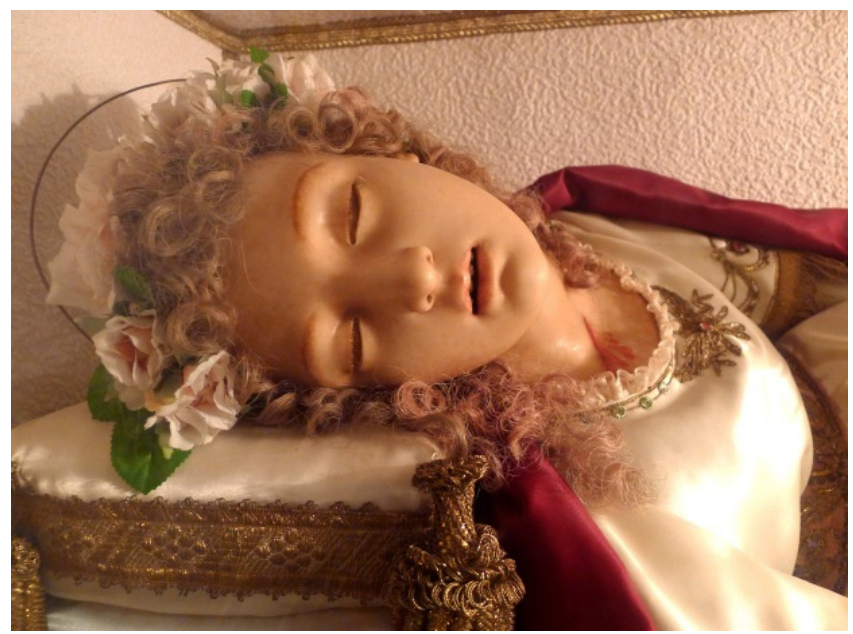

Figura 1.- Imagen de devoción. Santa Faustina, corpore santo (1829). Parroquia de San Juan Bautista (Pasai Donibane, Gipuzkoa). Imagen en cera de una niña yacente lujosamente vestida, alojada en un sarcófago de madera dorada y acompañada de relicario y autentica. El excepcional modo de presentación de esta reliquia constituye hoy en día su valor más significativo, si bien hasta hace unos años la devoción que suscitaba no era en absoluto desdeñable. Valoración: ALTO, 71. imprescindible para la gestión de los mismos, con el fin de garantizar su protección y clasificación en diversos niveles, identificar prioridades en la conservación, planificar operaciones de salvaguarda, etc.

El Centro de Patrimonio Cultural del Gobierno Vasco, en el curso de la renovación de su sistema de información documental ${ }^{3}$, encargó en 2013 a Albayalde-conservatio la reforma de la ficha de catalogación de bienes muebles y la inserción del campo de valoración cultural en vistas a su protección legal ${ }^{4}$. Ello conllevó el diseño de una herramienta que permitiera la clasificación de los bienes según su valor, a partir de la determinación de una serie de criterios y la cuantificación de los mismos 5 .

La aplicación del formulario resultante permite avanzar significativamente en la objetivación de un proceso que anteriormente constituía un ejercicio personal de subjetividad. A pesar de ello, debemos ser conscientes de que la valoración no es un acto totalmente objetivo y dependerá en parte del bagaje cultural, de los gustos y preferencias y del conocimiento del contexto del experto que lo aplique.

Por otra parte, y en el mismo sentido, es necesario reflexionar sobre el hecho de que las valoraciones no son definitivas. Al contrario, son cambiantes y dinámicas, al estar íntimamente ligadas a los valores de la sociedad y de las comunidades que la constituyen, variando en función de la evolución de los mismos.

\section{Metodología}

El primer paso para la puesta a punto del sistema fue la creación de un grupo de trabajo de expertos que aunara diversos perfiles, con la finalidad de abarcar tanto las numerosas tipologías de objetos como los diferentes aspectos a evaluar en un bien mueble. Se optó por un equipo reducido con la finalidad de agilizar los procesos y favorecer un contraste de opiniones dinámico.

Entre los participantes, firmantes de este artículo, figuran historiadores del arte especialistas en diversos periodos cronológicos o disciplinas artísticas, expertos en patrimonio inmaterial y profesionales con experiencia contrastada en la realización de inventarios y documentación ${ }^{6}$, poseyendo numerosas publicaciones en el terreno del patrimonio cultural. La inclusión de representantes de la administración pública y de la Iglesia ha resultado de vital importancia para la viabilidad del proyecto, puesto que aportaban una visión específica de la cuestión. El grupo ha contado con la asesoría del historiador Pedro Echeverría Goñi, destacado investigador y profesor de la Universidad del País Vasco.

El equipo, para la realización de su trabajo, se ha apoyado en las diversas experiencias acumuladas en la trayectoria profesional de sus miembros, en la literatura especializada 
sobre el tema y en acciones precedentes que presentaban afinidades con el proyecto en curso. Destaca entre ellas la llevada a cabo, en 2010, por el Museo Catharijneconvent (Utrecht), Museo Nacional de Arte Sacro de los Países Bajos, que lideró un proyecto destinado a establecer directrices para la ideación de un sistema de expurgo y reubicación de colecciones de objetos religiosos (Beyer y Takke 2012). Por otro lado, en 2009 el Collections Council australiano mejoró una herramienta ideada en 2001 para evaluar la importancia de sus colecciones en todo el país, con el fin de racionalizar los recursos dedicados a ello (Russell y Winkworth 2009). En 2012, a partir de un proyecto europeo, el Consejo de Europa editó una guía con una serie de directrices diseñadas para profesionales del campo de la protección del patrimonio, en respuesta a la situación provocada tras el conflicto de Kosovo y como ayuda para los países en desarrollo (Consejo de Europa 2012). En la misma dirección, la Ducht Cultural Heritage Agency (RCE) ha desarrollado un Manual en seis pasos para la evaluación de Colecciones de Museos (VV. AA. 2014).

A partir de estas ediciones y de las discusiones internas del grupo se definieron y calificaron los diferentes valores atribuibles a un bien mueble del patrimonio religioso, asignándoles una puntuación en función de la importancia estipulada. Tras ello, se diseñó un formulario que nos permitiera una aplicación relativamente sencilla. Para su estructura general nos hemos basado en sistemas de valoración de bienes inmuebles, con una trayectoria más prolongada en este tipo de procesos ${ }^{7}$.

\section{Patrimonio cultural mueble}

Bajo el concepto de "Patrimonio cultural mueble" se integran objetos de muy diferente naturaleza, origen y función. La amplitud de su definición tiene como primera consecuencia el abundante número de piezas que pueden ser consideradas como tal y, a partir de ahí, la dificultad para establecer inventarios completos y con una visión global que permita su justa evaluación [figura 2].

La propia condición de bien mueble, determinada por su movilidad, obstaculiza su control puesto que a menudo están expuestos a traslados, pérdidas, ventas o robo. Cuando no está custodiado en los museos, puede ser un patrimonio escasamente conocido y por lo tanto poco protegido. En edificios religiosos frecuentemente están sujetos a cambios de gusto, reformas y manipulaciones, intervenciones no profesionales, cambios de ubicación, etc.

La primera cuestión a la que nos enfrentamos a la hora de abordar el sistema de evaluación es la siguiente: ¿Se puede realmente establecer criterios suficientemente generalistas que sean útiles para una cantidad de bienes de tipología tan diversa? O al contrario, ¿se debería valorar cada tipo de elemento por separado y así poder precisar más en valores y cuantificaciones?

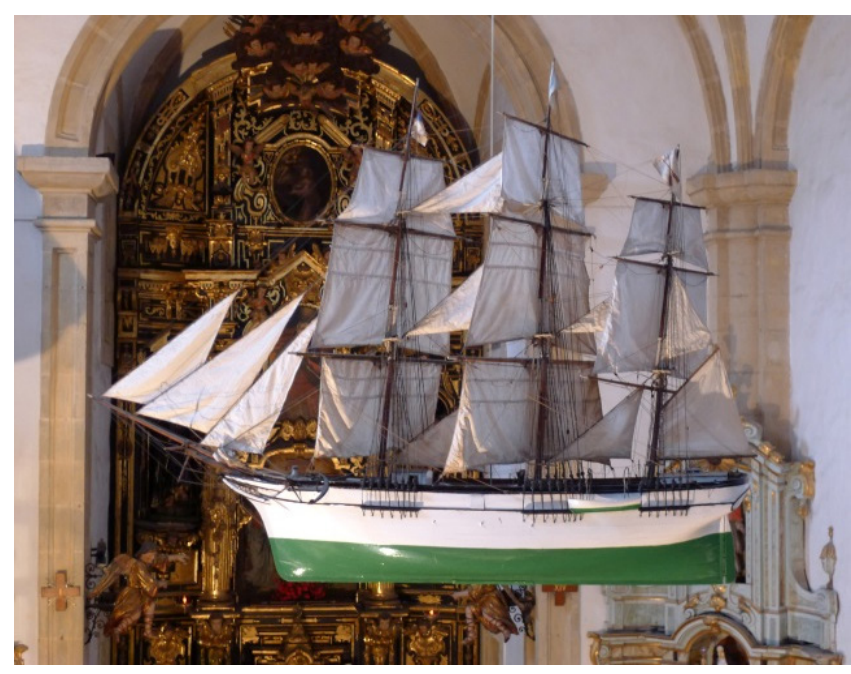

Figura 2.- Exvoto marinero que cuelga de la bóveda de la iglesia, s. XX, Francisco Tuduri, Basílica del Santo Cristo de Bonanza (Pasai Donibane, Gipuzkoa). La fragilidad de este tipo de obras requiere una especial consideración. Esta maqueta es un ejemplar digno y bien conservado de modelismo naval y representa la tradición del uso de estos elementos como objetos de ofrenda. Valoración: ALTO, 59.

Dada la gran abundancia en número de los bienes muebles y la diversidad de los mismos, es poco realista pensar en la aplicación de un sistema demasiado complicado, con múltiples variantes. En aras del sentido práctico y de la creación de una herramienta que realmente sea útil, se han primado procedimientos sencillos que proporcionen cierta agilidad, por lo que se han elegido valores que puedan caracterizar distintos tipos de objetos.

\section{Valores}

La recopilación de valores se hallevado a cabo tras sopesar los conceptos preseleccionados y adaptarlos a las necesidades impuestas por el proyecto piloto a realizar ${ }^{8}$. La mayor dificultad ha residido precisamente en la restricción voluntaria de dichos valores a un número reducido y en la definición de cada uno de ellos, cuando en la realidad a menudo sus límites son difusos, se hallan combinados entre ellos, son acumulativos y en algunos casos incluso contradictorios ${ }^{9}$.

Riegl distinguió dos grandes grupos de valores: los valores Rememorativos (de antigüedad, histórico y conmemorativo) y los de Contemporaneidad (instrumental y artístico) (Riegl 1999). Mason a su vez diferencia entre valores Socioculturales (histórico, cultural-simbólico, social, espiritual/religioso y estético) y Económicos (valor de uso -mercado- y valor de no-uso -no-mercado-) ${ }^{10}$; en la misma línea, Szmelte propone una estructura de evaluación basada en dos categorías principales: valores CulturalesHistóricos y valores Socio-Económicos, que aglutinan respectivamente numerosos subcriterios ${ }^{11}$ (Szmelte 2013). Beyer y Takke añaden a los Valores Actual e Histórico, los Criterios Comparativos (excepcionalidad, conservación, 
Tabla 1.- Resumen de los valores y subvalores de un objeto de patrimonio mueble.

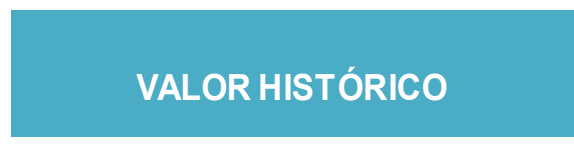

\begin{tabular}{|c|}
\hline ANTIGÜEDAD \\
\hline \hline TESTIMONIAL \\
\hline \hline DOCUMENTACIÓN \\
\hline \hline REFERENCIAL \\
\hline
\end{tabular}

VALOR MATERIAL

\begin{tabular}{|c|}
\hline NATURALEZA \\
\hline \hline INTEGRIDAD \\
\hline \hline CONSERVACIÓN \\
\hline VULNERABILIDAD \\
\hline
\end{tabular}

de conjunto, de presentación y documental) ${ }^{12}$ (Beyer y Takke 2012: 34-39), lo que les permite matizarlos; mientras que la Dutch Cultural Heritage Agency (RCE) plantea cuatro categorías: Características (conservación, conjunto, proveniencia, excepcionalidad y representatividad), Valor Histórico-cultural (histórico, artístico, de información), Social y "Societal" (social, de percepción), y de Uso (museístico, económico), añadiendo un quinto apartado denominado Adicional, para otros criterios que pudieran surgir en el curso de la evaluación ${ }^{13}$ (VV.AA. 2014: 23-48).

De este resumen se desprende que, aunque hay una evidente coincidencia en la caracterización de ciertos valores y criterios, así como una diferencia entre aquellos más actuales y los considerados históricos, no se observa por el momento un consenso claro en la forma de agruparlos, combinarlos o calificarlos, que es heterogénea y múltiple. En este sentido creemos importante la reflexión de la Dutch Cultural Heritage Agency, que expone la necesidad de flexibilizar los sistemas de evaluación y adecuarlos a cada caso en concreto (VV.AA. 2014: 4-5).

En este proyecto piloto se ha preferido la estructura sencilla de cinco valores independientes (con sus consiguientes subvalores), sin reagrupamientos entre ellos o asignación de rol comparativo con respecto a los demás. Estos son: Valor Histórico, Valor Artístico, Valor Social/Espiritual, Valor Material y Valor de Conjunto [tabla 1].

\section{Valor Histórico}

—Antigüedad ${ }^{14}$ : la Antigüedad es un valor por sí mismo, ya que el paso del tiempo destruye, hace desaparecer y

\section{VALOR DE CONJUNTO}

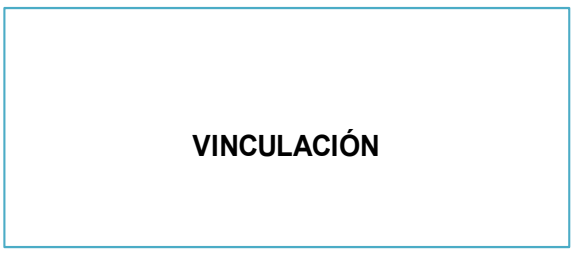

Figura 3.- Santa Ana y la Virgen, s. XVII, talla policromada, Ermita de Santa Ana (Pasai Donibane, Gipuzkoa). Grupo escultórico de la Educación de la Virgen compuesto por dos figuras de bulto redondo. Las tallas poseen una notable calidad, tanto en la caracterización de los rostros como en el tratamiento de las telas que les dota de gran monumentalidad y dinamismo. Valoración: ALTO, 70. 
transforma los objetos muebles. Independientemente de su valor artístico, técnico o simbólico, el hecho de que un objeto antiguo llegue hasta nuestros días constituye un valor por sí mismo. Es el valor más objetivo y por ello probablemente el principal de todos ellos. Nos remonta a épocas donde el objeto en sí puede ser el único documento existente, la única fuente de información sobre sí mismo o su contexto [figura 3].

-Testimonial: ciertos objetos poseen un valor histórico general, bien porque están conectados con un importante evento, grupo, figura o lugar, bien porque ilustran la historia local o regional o ayudan a comprender la vida cotidiana de una época o lugar, bien porque la sociedad les atribuyó un valor en el momento de su creación.

—De documentación: si una pieza está documentada a partir de contratos, referencias, escritos de archivo, dibujos o bocetos o estudios posteriores que aportan conocimiento sobre la misma o sobre su época, cuenta con un valor añadido. Si esta información suplementaria constituye una aportación desde el punto de vista de la investigación cultural o histórica, el valor aumentará.

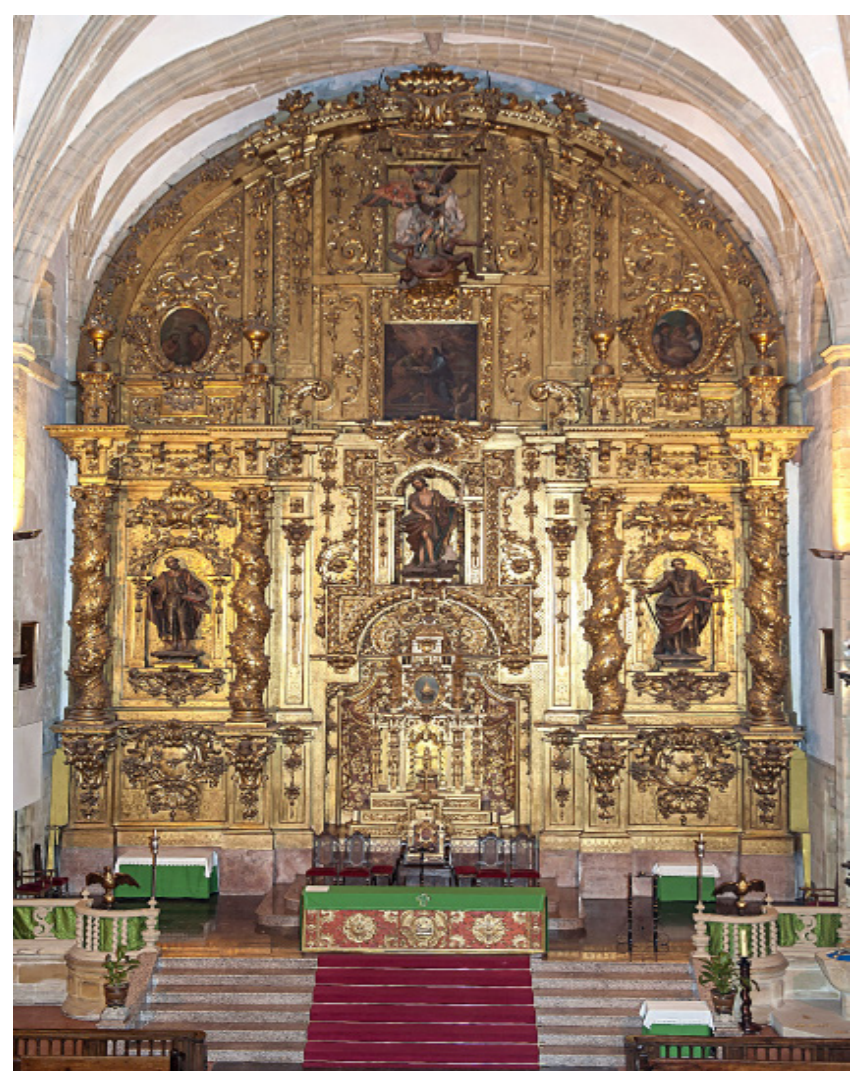

Figura 4.- Retablo mayor de San Juan Bautista, s. XVIII, Sebastián de Lecuona. Pintura: Felipe de Arizmendi, Parroquia de San Juan Bautista (Pasai Donibane, Gipuzkoa). Es un mueble de gran tamaño que se ajusta a las características del retablo churrigueresco y en el que intervienen destacados artistas como Lecuona o Arizmendi dando lugar a una obra de apreciable calidad. Cea Bermúdez incluía la talla del titular entre las más notables de Felipe de Arizmendi. Valoración: ALTO, 74.
-Referencial: Un objeto es importante también si constituye una referencia con respecto a otros, es decir, si a partir de él se pueden establecer atribuciones, relaciones o es posible reconstruir un contexto.

\section{Valor Artístico}

—Relevancia de autoría/producción: para aquellos objetos realizados por un artista, taller, escuela o en un entorno artístico, considerados importantes en el campo de la historia del arte o de la producción o para los que reflejan el talento artístico o la creatividad. Esta categoría no se limita a obras destacadas, sino que obras de arte de menor calidad pueden ser de interés al ilustrar el desarrollo de un artista [figura 4].

- Calidad técnico/estética: un objeto se considera que posee calidad técnica o estética si su diseño, forma, revestimiento, etc. refleja un alto estándar de fabricación. El diseño y ejecución, la habilidad, la precisión o el esmero en su proceso de producción, su plasmación en un objeto estéticamente satisfactorio y armonioso son significativos a este respecto. Los bienes no tienen por qué ser obras de arte para tener calidad. Un instrumento puede ser juzgado a nivel de su aspecto artístico, de fabricación y también por la calidad de su sonido o de su "maquinaria", es decir, a nivel técnico.

—Singularidad: para aquellos objetos únicos, originales o raros.

-Representatividad: el objeto ilustra una tendencia específica y reúne un conjunto de características determinadas que lo definen como "tipo" o "modelo", a partir del cual se ha desarrollado una tipología reproducida por otros autores o talleres.

\section{Valor Social}

-Identitario: cuando el bien agrupa una serie de valores intangibles, como el de pertenencia a una comunidad, valor simbólico, emocional, devocional, conmemorativo o de su historia particular. Son objetos representativos de una colectividad, testimonio de ella y de sus valores propios, sociales, espirituales $y / 0$ religiosos ${ }^{15}$. Se asocia en este último ámbito a objetos venerados por sus propiedades devocionales como las reliquias y las esculturas de santos [figura 5].

-Continuidad de su uso: se considera que el mantenimiento del uso o función original para el que fue fabricado un objeto representa un valor añadido, ya que la colectividad le sigue dando importancia [figura 6].

-Educativo: se atribuye a aquellos objetos capaces de suscitar el interés público o/y poseer un valor para la formación. Pueden ser adecuados para su exhibición, para 


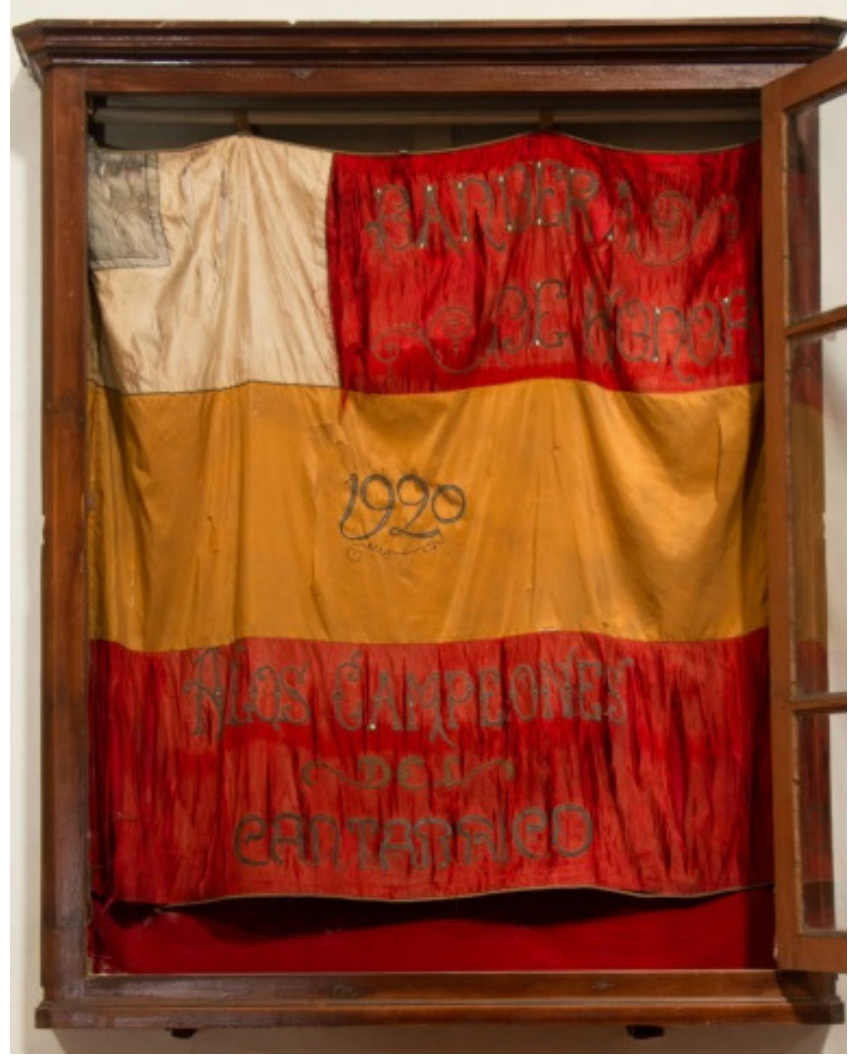

Figura 5.- Bandera de la Concha, 1920, Basílica del Santo Cristo de Bonanza (Pasai Donibane, Gipuzkoa). Bandera de España que presenta en la parte superior la enseña de Donostia/San Sebastián. Es una pieza que recoge la tradición de regatas en el Cantábrico y la participación de Pasaia en las mismas, con la victoria en dicho año. El hecho de encontrarse custodiada en la Basílica demuestra la interrelación entre actividades de orden civil y religioso en esta comarca durante este periodo. Valoración: ALTO, 58.

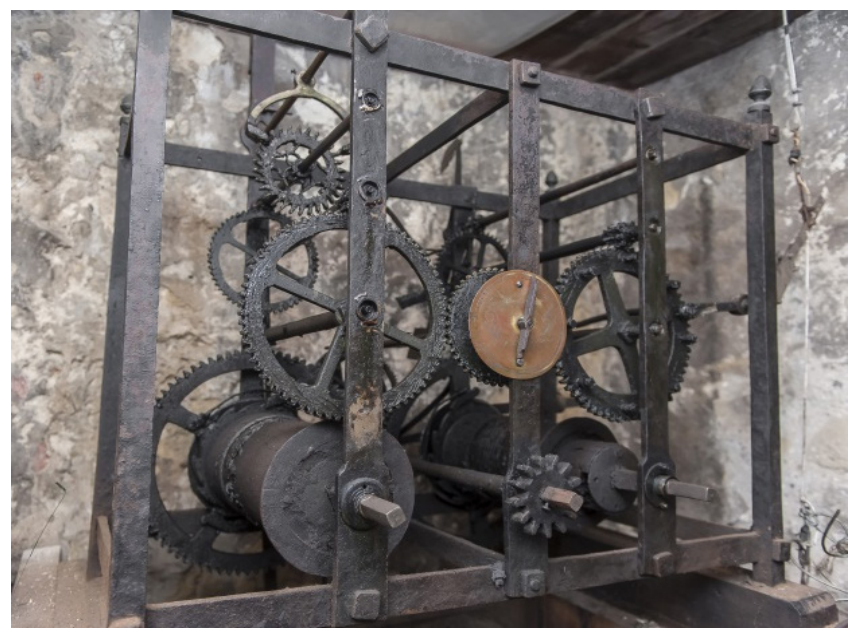

Figura 6.- Reloj-campana, s. XIX, Ermita de Santa Ana (Pasai Donibane, Gipuzkoa). Reloj mecánico de hierro forjado de fabricación artesanal, obra de un miembro de la familia Zugasti, saga de relojeros activa desde principios del siglo XIX que realizó más de una treintena de relojes públicos en el País Vasco. La conservación íntegra del conjunto, compuesto por la maquinaria, pesas y campana, y el hecho de estar en funcionamiento con su sistema original, incrementan su valor. Valoración: ALTO, 74.

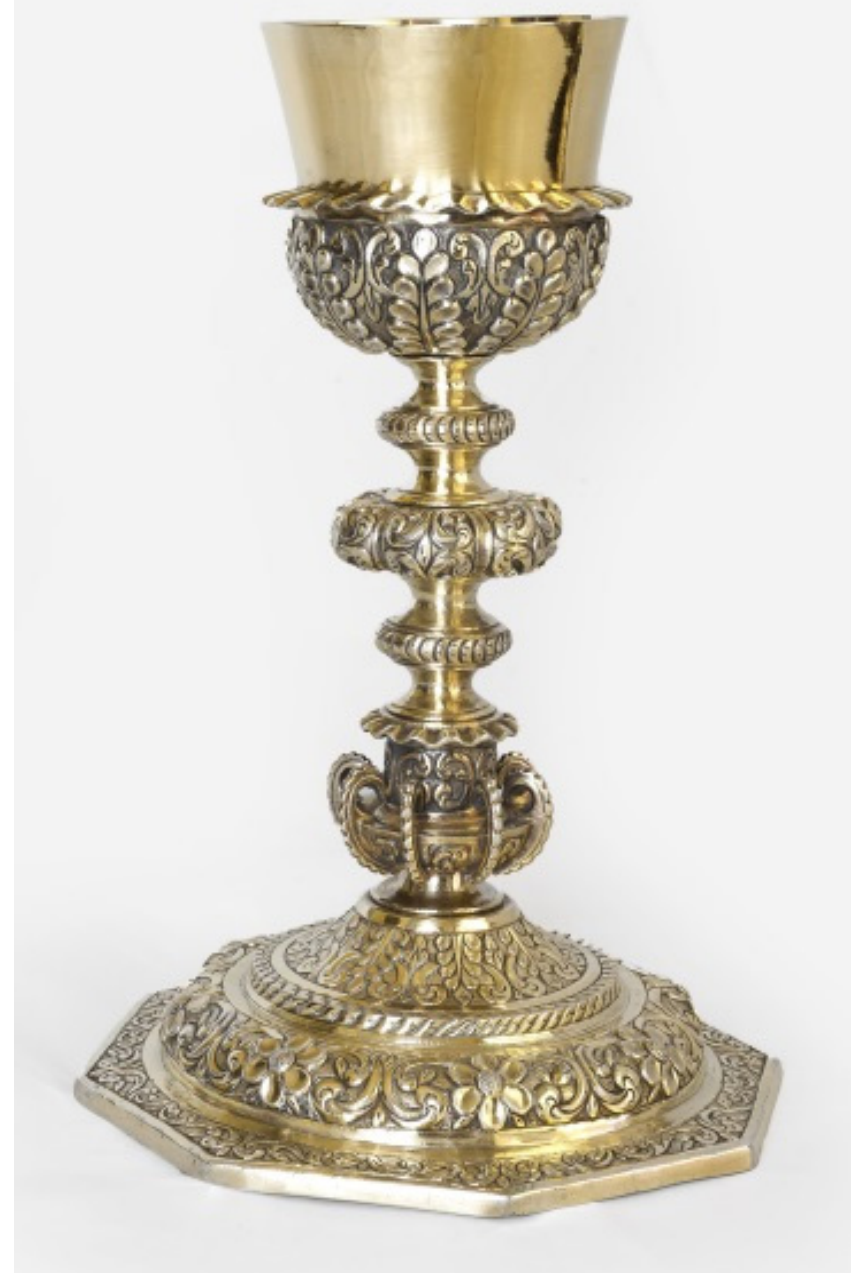

Figura 7.- Cáliz, $2^{\circ}$ cuarto del s. XVIII, Parroquia de San Juan Bautista (Pasai Donibane, Gipuzkoa). Cáliz de base octogonal recubierto de una rica decoración de elementos vegetales. De gran calidad en su factura, es una de las pocas piezas de origen guatemalteco que se han conservado en el territorio e incluso en España. Valoración: EXCELENTE, 80.

formar parte de programas didácticos, divulgativos, etc., para la transmisión de mensajes o para la interpretación de contextos.

\section{Valor Material}

- Naturaleza: calidad y singularidad de los materiales nobles o excepcionalmente utilizados por su elevado precio, lugar de origen, dificultad de extracción, etc. [figura 7].

—Integridad: un objeto puede ser importante en razón de su buen estado de conservación, por la ausencia de alteraciones significativas y la permanencia de sus materiales y diseño originales. En ese sentido puede despertar en las personas que lo contemplan una sensación de "autenticidad"16. 
- Conservación: el grado de conservación condiciona el valor de un objeto y puede ser decisivo, pudiendo graduarse: bueno $=$ sin daños o deterioro, objeto estable. No precisa conservación ni restauración; regular = daños menores que no desvirtúan el bien ni en su aspecto material ni en su significado. Puede requerir ciertas tareas de conservación preventiva o curativa; malo = daños limitados que desvirtúan moderadamente el objeto. Presenta poca estabilidad y/o el deterioro está activo. Puede precisar tareas de conservación o restauración; pésimo $=$ con daños importantes, no es posible su utilización, el objeto está en peligro, con un deterioro activo importante $y / o$ se requiere una intervención de restauración con urgencia.

-Vulnerabilidad: fragilidad que puede hacer que el elemento desaparezca. Se mide en un contexto donde otros de sus características se han perdido. Se trata de un factor intrínseco, debido a las propias características: naturaleza de sus materiales, forma, sensibilidad a factores externos, etc.

\section{Valor de Conjunto}

Este valor se reconoce cuando el objeto pertenece a un conjunto, a una colección o bien está vinculado con un inmueble, patrimonio inmaterial, sitio histórico, etc. Las razones de una vinculación entre objetos o con otros bienes patrimoniales pueden ser diversas: históricas, artísticas o sociales (contemporaneidad de producción, localización, relación con personas, actos o lugares, unidad estilística, tipológica, relevancia, etc.).

En general, podríamos afirmar que la separación de un objeto de su conjunto conlleva una devaluación del valor, significado o cualidades, tanto del objeto en sí mismo como del conjunto afectado.

\section{Tabla cuantitativa}

Una vez fijados los criterios o valores, es imprescindible establecer una baremación de los mismos, de manera que sea posible una "puntuación", aunque sea relativa, del objeto. Este registro puede llevarse a cabo de forma verbal o numérica ${ }^{17}$. Para la primera hemos asignado cuatro parámetros o niveles: excelente, alto, medio o bajo, calificando el bien dentro de una de esas categorías. Para la segunda, se determinan valores numéricos, distribuidos también en cuatro grupos que se corresponden con los valores verbales.

La baremación verbal resulta menos precisa, ya que no es posible especificar más allá que la inclusión dentro del nivel de un grupo determinado. Si el objeto es "excelente", desconocemos si es un excelente alto o un excelente bajo. La baremación numérica, en cambio, es más rigurosa y permite clasificaciones más exactas, pudiendo señalar qué objetos dentro de una misma categoría son superiores a otros, con simples operaciones matemáticas. Sin embargo, en la práctica y en el cómputo total de valores, a veces, refleja una cierta arbitrariedad menos presente en la calificación verbal que se revela como más justa. En realidad ambas son complementarias, y de su cotejo se obtiene un perfil más adecuado del objeto, razón por la cual incluimos ambas en el formulario.

Para poder llevar a cabo la cuantificación correctamente, se debe otorgar un valor a cada criterio y subcriterio, ya que no todos tienen el mismo alcance ni trascendencia. Así, se han primado como más significativos dentro de cada grupo los subvalores de antigüedad, calidad, identidad e integridad.

Para establecer la graduación hemos dividido los objetos en dos grandes grupos, en función de su tipología. El primero reúne los bienes de bellas artes y artes aplicadas $y$, en general, todos aquellos en los que la calidad de su elaboración y una clara voluntad artística o decorativa predomina sobre su función. La segunda familia está integrada por aquellos objetos de tipo etnográfico (a falta de una mejor calificación), poseedores de una menor calidad o relevancia artística o estética ${ }^{18}$. El formulario a utilizar es el mismo en los dos casos, cambiando únicamente la puntuación máxima de cada apartado. Así, se otorgará un peso mayor al valor más específico y definitorio: el valor artístico para el primer grupo (30/100); el valor social/de identidad para el segundo (30/100). El resultado final es el formulario adjunto [tabla 2].

En la parte superior de la ficha se identifica el bien con una serie de datos básicos (denominación, número de inventario o registro e inmueble donde está ubicado). En el apartado de valores se va calificando cada uno de ellos: primero de forma verbal, seguidamente con números, estableciendo una valía dentro del máximo atribuible.

El valor absoluto del objeto resulta de la suma de los diferentes valores (baremación numérica) y de la extrapolación de la media de las calificaciones adoptadas (baremación verbal).

En la zona lateral derecha del formulario, la sección de observaciones permite justificar la elección realizada, de gran interés para comprender la importancia y el significado del bien.

\section{Resultados del proyecto piloto}

La ficha ha sido testada en un programa piloto de catalogación en tres edificios de diferente categoría y naturaleza (una basílica, una parroquia y una ermita) de Pasai Donibane (Gipuzkoa). De los ciento trece elementos catalogados se han considerado treinta y cuatro como bienes muebles meritorios de ser protegidos legalmente por su valor cultural: tres con el nivel de EXCELENTE y 
Tabla 2.- Formulario de valoración

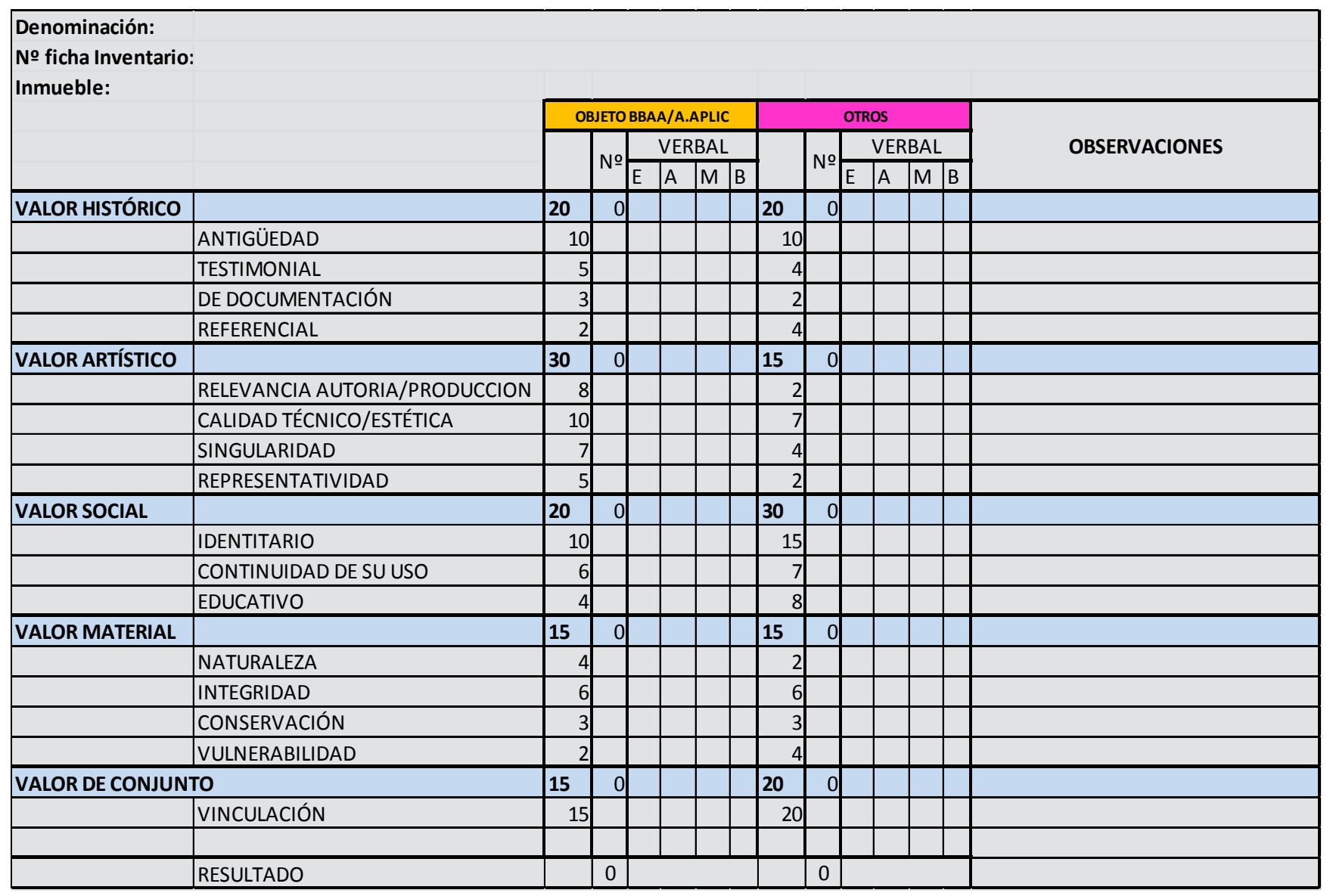

treinta y uno con ALTO. Dentro de los primeros, se hallan dos tallas policromadas ${ }^{19}$ (s. XVI y XVII) y un cáliz (s. XVIII), mientras que entre los segundos, se contemplan cuatro retablos ${ }^{20}$, tres tallas policromadas ${ }^{21}$, dos pinturas sobre lienzo ${ }^{22}$, diez piezas de platería ${ }^{23}$, un púlpito (s. XVII), una reja (s. XVIII), un mausoleo (s. XIX), un frontal de altar (s. $\mathrm{XVIII),} \mathrm{un} \mathrm{corpore} \mathrm{santo} \mathrm{(s.} \mathrm{XIX),} \mathrm{un} \mathrm{reloj-campana} \mathrm{(s.} \mathrm{XIX),}$ una exvoto en forma de maqueta de barco $(X X)$ y dos estandartes (s. XX). Prácticamente en todos ellos había un fuerte componente artístico y/o histórico, si bien el valor social ha sido determinante en la estimación de los cinco últimos, por su uso o la significación emocional para la población. En todos ellos el valor de conjunto, bien por estar vinculados a un grupo de objetos o al inmueble que los cobija, ha sido muy influyente en la puntuación.

De manera general, podría concluirse que el manejo del formulario ha resultado satisfactorio en esta primera campaña. A las dudas iniciales ha sucedido una clara comodidad en su utilización. Ciertos valores han sido afinados, con correcciones y observaciones tanto de contenido (al no estar precisados suficientemente los valores o solaparse entre ellos) como de cuantificación (la suma de valores daba una valoración final desacertada). En ocasiones los resultados verbales y numéricos no coincidían con exactitud y en estos casos se ha optado por privilegiar la puntuación verbal y los argumentos narrativos, más justos. Los expertos se han visto confrontados a evaluar separadamente y con escrupulosidad valores que anteriormente contemplaban en su conjunto, lo que ha procurado un enriquecimiento en las justificaciones. Una labor última de reajuste entre todas las valoraciones ha sido necesaria para equilibrar el conjunto.

Con todo, el esfuerzo de analizar cada uno de los registros imponía cierto desapego de los propios gustos y evitaba la tendencia natural a olvidar los aspectos más ajenos a las propias inclinaciones. En ese sentido, la objetividad ha resultado beneficiada. El uso sin embargo ha sido muy limitado. En experiencias posteriores esta herramienta deberá ser contrastada con un número superior y a una mayor variedad de bienes. Su empleo en próximas operaciones, todavía sin perfilar, permitirá afinarla y juzgar su viabilidad como instrumento de valoración.

\section{Notas}

[1] La bibliografía que aborda de manera íntegra o parcial la definición e incluso la clasificación de las cualidades o valores de los bienes culturales es considerable. Autores como Riegl (Riegl 1903), Baudrillard (Baudrillard 1975), Avrami, Mason y de la Torre (Avrami 2000, Mason 2002) o Sztelme (Sztelme 2013) entre otros se enfrentan al reto de responder a la pregunta 
¿qué caracteriza al patrimonio cultural? En la misma dirección han trabajado entidades dedicadas a la gestión y conservación del patrimonio cultural - Parks Canada, Getty Conservation Institute, English Heritage, Dutch Cultural Heritage Agency, Australia Government, etc.-.

[2] Como proyectos prácticos de evaluación de bienes culturales muebles aplicados a un fin específico, destacan los elaborados en Holanda: el Plan Delta (1989-2000) (Kirby Talley Jr 1999); el Proyecto de inventario de museos MUSIP (1997-1999); la Guía para la Valoración Cultural de Interiores Históricos (Broker, Hartog, Huisman y Luger 2011); el Proyecto de Evaluación de Objetos Religiosos (2011) (Beyer y Takke 2012) o la Evaluación de Objetos de Museos (VV. AA. 2014).

[3] El Gobierno vasco ha migrado recientemente la documentación comprendida en sus diversos inventarios o catálogos en un sistema de información patrimonial denominado INTEGRA. Se trata de una plataforma informática con vistas a facilitar la organización, gestión y control de la red de información del Centro de Patrimonio Cultural Vasco así como la gestión del Registro de Bienes Culturales Calificados y del Inventario General de Patrimonio Cultural Vasco.

[4] El Estatuto de Autonomía del País Vasco reconoce como competencias exclusivas de la Comunidad Autónoma la cultura, el patrimonio histórico y los archivos, bibliotecas y museos, salvo los de titularidad estatal. Ley 7/1990, de 3 de Julio, de Patrimonio Cultural Vasco, Exposición de motivos.

[5] No es la primera experiencia de este tipo que se lleva a cabo en la Comunidad Autónoma del País Vasco. Echeverría Goñi lideró en 2000-2001 una operación de protección que tuvo como consecuencia la declaración como Bienes Culturales Calificados (máximo nivel de protección en esta comunidad) de 71 retablos o conjuntos, entre los 3000 ejemplares que se calcula existentes en estos territorios. En la publicación editada en esa ocasión, el historiador expone los criterios generales que han guiado su elección (Echeverría y Arechaga 2001). Por otra parte, la Fundación Zain, dedicada principalmente a proyectos de innovación tecnológica de gestión integral del patrimonio, realizó en 2010 un plan piloto de valoración de caseríos de Elorrio (Bizkaia), diseñando un cuadro detallado de evaluación de datos (Inédito).

[6] I. Migueliz participó en la elaboración de la Base de Datos e Imágenes del Catálogo Monumental de Navarra (2001-2005), del Catálogo de Bienes Muebles del Patrimonio Cultural de Navarra (2006-2010) y del Plan Director de la Catedral de Pamplona. J. Muñiz formó parte del equipo responsable de los Inventarios de Arquitectura Neoclásica y de Arquitectura y Escultura Funeraria en el País Vasco (1999-2001) y del de Valoración de Retablos anteriormente citado. Realiza tareas de documentación de la colección del Museo Diocesano de Arte Sacro de Bilbao desde 2002 y es autor del estudio sobre escultura tardogótica mueble en Bizkaia que incluye un inventario de piezas. M. P. Timón es coordinadora del Plan Nacional de Salvaguarda del Patrimonio Cultural Inmaterial, del Plan
Nacional de Arquitectura Tradicional y del Plan Nacional de Abadías, Conventos y Monasterios.

[7] En efecto, como veremos más adelante, la evaluación en bienes muebles adopta en algunos formularios sistemas comparativos relativamente complejos de utilizar (Beyer y Takke 2012, Consejo de Europa 2012). En cambio, las valoraciones de edificios muestran en la mayor parte de los casos una estructura más lineal y de fácil aplicación (Kalman 1980, Angel 2009)

[8] Estas necesidades se resumen básicamente en tres puntos 1 /Valores asignables a bienes muebles y ubicados en edificios de carácter religioso 2/ Formulario fácilmente gestionable en el curso de la revisión un inventario 3/ Resultado que facilite una posterior agrupación de bienes según diversas categorías para una posible protección legal.

[9] Es la idea expresada por R. Mason: "While the subjectivity and contingency of heritage values make it difficult to establish a clear framework or even a nomenclature of values ...this is precisely what is needed to facilitate the assessment and integration of different heritage values in conservation planning and management. So the concept of values needs to be broken down and defined in a typology, at least provisionally" (Mason 2000: 9).

[10] Sociocultural Values: historical, cultural /symbolic, social, spiritual/religious and aesthetic. Economic Values: use value (market value), nonuse value (nonmarket value) (Mason, 2002).

[11] Cultural-Historical Values: relative artistic, aesthetic (visual appeal) and age value, historical, identity, scientific, rarity, authenticity, emotional, integrating, associative/symbolic and creative. Contemporary Socio-Economic Values: educational, economic, functional, social, social access, political, operational, newness, situational, financial, potential.

[12] Current values, Historical values (Church historical value, general historical value, art-historical value) and Comparative criteria (rarity, condition, ensemble value, presentation value, documentary value)

[13] Features: Condition, ensemble, provenance, rarity and representativeness; Culture historical: historical, artistic, information value ; Social and Societal: social y perception; Use: museum y economic: Additional

[14] Echeverría y Arechaga detallan los criterios que han sido adoptados en la valoración de retablos: antigüedad, excepcionalidad, sentido devocional y características de los materiales. Así, en relación al primero de ellos afirman: "los objetos que sobreviven a tal acción [la del tiempo] presentan, con independencia de otros valores como la calidad, un peso específico dentro del patrimonio de un lugar. Así, la antigüedad para los retablos de fines del siglo XV y primer tercio del siglo XVI supone motivo suficiente para ser seleccionados" (Echeverría y Arechaga 2001:415). 
[15] A propósito de la generación de identidades, Muñoz escribe: "La función identitaria también ha sido señalada por muchos autores como inherente al patrimonio cultural (cfr., p. ej, Morente 2001; Ballart, 1996 o MacLean, 1995), aunque es necesario tener presente que la función de los objetos no es generar identidades sino simbolizarlas, representar unas identidades que han sido generadas con anterioridad, que preexisten al símbolo; los símbolos contribuyen a su continuidad, pero no la generan". Y continua con respecto a los objetos simbólicos: "Para aquellos que desconocen los códigos y las circunstancias de otras comunidades o personas, estos valores no existen o resultan incomprensibles o ridículos" (Muñoz 2003: 47,49).

[16] El concepto de autenticidad ha evolucionado de forma considerable en los últimos años. El documento de Nara (ICOMOS, 1994) expuso su nueva significación, entendida no ya como una mera preservación de los valores materiales de un objeto en un supuesto estado original, sino también como la perduración de sus atributos más intangibles (idea, mensaje, función, etc.). Aquí la noción de autenticidad viene asimilada de manera más limitada a "integridad" y "originalidad", estando los aspectos inmateriales recogidos dentro de Valor Social.

[17] Por poner un ejemplo, en el proyecto de Evaluación de Objetos Religiosos (2011) (Beyer y Takke 2012) utilizaron únicamente la valoración verbal, pues la numérica no les aportaba resultados satisfactorios. Para la valoración de un objeto, en un primer paso califican el valor actual y el valor histórico: alto, medio, bajo, no aplicable, estableciendo la media entre ellos. Este resultado provisional es ajustado mediante los criterios comparativos, que la pueden aumentar o reducir, aunque no cambiarla substancialmente.

[18] En un primer momento, los bbmm se dividieron en tres grandes grupos, añadiendo a los actuales un apartado específico para instrumentos musicales. Este formulario independiente fue desechado posteriormente ya que no aportaba cambios substanciales con respectos a los dos anteriores.

[19] Cristo de Bonanza de Jerónimo de Larrea (s. XVI) y Santa Ana y la Virgen, de autor anónimo (s. XVII).

[20] Retablo mayor del Santo Cristo de Bonanza (s. XVIII), retablo mayor de San Juan Bautista (s. XVIII), realizado por Sebastián de Lecuona y con pinturas de Arizmendi y el conjunto formado por los retablos colaterales de San José y San Francisco de Borja (s. XVII).

[21] San Juan Bautista (s. XVII) y una pareja de ángeles lampadarios (s. XVIII).

[22] La Degollación del Bautista (s. XIX) y San Carlos Borromeo (s. XVII), de autor desconocido.

[23] Dentro de platería, un conjunto de vinajeras, custodia y cáliz de José María Rodallega (s.XIX), un conjunto de incensario y naveta de Ignacio Larrañaga (s. XVIII), tres cálices (s. XVI y XVIII), una vinajera (s. XVIII, José Legarda), una demanda (s. XVIII, Ignacio Larrañaga), un copón (s. XVII, Martín Arano de Zumarrista), una taza (s. XVII) y una cruz procesional (s. XVIII).

\section{Bibliografía}

ANGEL, V. (dir.) (2009). Manuel de référence du Bureau d'examen des Édifices Fédéraux du Patrimoine. Québec: Bureau d'examen des édifices fédéraux du patrimoine (BEÉFP).

AVRAMI, E., MASON R., and TORRE, M. (eds). 2000. Values and Heritage Conservation, Los Angeles: Getty Conservation Institute.

BAUDRILLARD, J. [1968] (1978). Le système des objets. Paris: Gallimard.

BEYER M. y TAKKE, J., 2012. Guidelines on ways of dealing with religious objects. Utrecht (Holanda): Museum Catharijneconvent

ECHEVERRÍA GOÑI P.L. y ARECHAGA ALEGRÍA S. (2001). "Criterios de valoración". En Erretaulak/Retablos, Echeverría Goñi P.L (dir.), Vitoria-Gasteiz: Gobierno Vasco. 411-427.

ICOMOS (1994). “Documento de Nara sobre la Autenticidad. Comité Español del Consejo Internacional de Monumentos y Sitios. http://www.esicomos.org/Nueva_carpeta/info_DOC_ NARAesp.htm [consulta: 15/05/2016]

KALMAN, H., 1980, The Evaluation of Historic Buildings. Ottawa: Environment Canada Parks Service.

KIRBY TALLEY JR, M. (1999). “El Plan Delta: una operación de salvamento a escala nacional", Museum, 201:11-16.

MASON, R. (2002). "Assessing Values in Conservation Planning: Methodological Issues and Choices". En DE LA TORRE (ed.) Assessing the Values of Cultural Heritage. Research Report. Los Angeles: The Getty Conservation Institute. 5-30.

MUÑOZ VIÑAS, S. (2003). Teoría contemporánea de la Restauración. Madrid: Editorial Síntesis

RIEGL, A. [1903] (1999). El culto moderno a los monumentos. Madrid:Visor.

RUSSELL R. Y WINKWORTH K., 2009. Significance 2.0., A guide to assessing the significance of collections. Adelaida: Collections Council of Australia.

SZMELTER, I. (2013). "New Values of Cultural Heritage and the Need for a New Paradigm Regarding its Care», en CeROArt. http://ceroart.revues.org/3647. [consulta: 09/09/2014] 
V. AA. (2011). Hulpmiddel bij de waardering van historische interieurs. Amersfort (Holanda): Rijksdients voor het Cultureel Erfgoed.

V. AA. (2012). Guidelines on Cultural Heritage. Technical tools for heritage conservation and heritage, JP - EU/CoE Support to the Promotion of Cultural Diversity (PCDK). Estrasburgo (Francia): Consejo de Europa.
VV.AA. ( 2013). Assessing cultural heritage significance. Using the cultural heritage criteria. Brisbane: Heritage Branch, Department of Environment and Heritage Protection (EHP). Queensland Government.

V. AA. (2014). Assessing Museum Collections. Collection valuation in six steps. Amersfoort (Holanda): Dutch Cultural Heritage Agency (RCE).

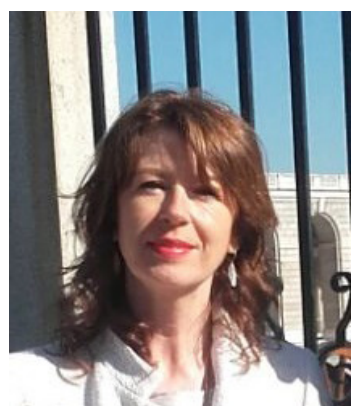

\section{Maite Barrio Olano}

Albayalde Conservatio mbarrio@albayalde.org

Maite Barrio Olano es directora de ALBAYALDE-conservatio desde 1989, empresa dedicada a la conservación del patrimonio cultural (www.albayalde.org).Trabaja principalmente para el sector público realizando numerosas intervenciones en el campo de la conservación preventiva y restauración; diseño de reservas de colecciones (Gordailua, Disseny HuB Barcelona, Museo de Colecciones Reales de Madrid); proyectos de investigación (POLICROM) y de difusión (Retablos Flamencos en España); proyectos europeos (Policromia) y de colaboración inter-institucional (Capilla Real de Granada). Licenciada en $\mathrm{H}^{\mathrm{a}}$ del Arte y Arqueología (Sorbona), licenciada en Conservación de Bienes Culturales (Panteón Sorbona), diplomada en Conservación y Restauración de Obras de Arte y Museología (UIA, Florencia), con estadía de formación en el Instituto Real de Patrimonio Artístico de Bruselas.

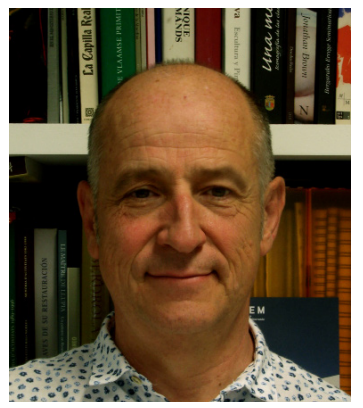

\section{Ion Berasain Salvarredi}

Albayalde Conservatio

iberasain@albayalde.org

Ion Berasain Salvarredi es jefe de proyectos y co-fundador de ALBAYALDE-conservatio, empresa dedicada a la conservación del patrimonio cultural (www.albayalde.org). Trabaja principalmente para el sector público realizando numerosas intervenciones en el campo de la conservación preventiva y restauración; diseño de reservas de colecciones (Gordailua en Irún, Disseny Hub Barcelona, Museo de las Colecciones Reales de Madrid); proyectos de investigación (POLICROM) y de difusión (Retablos Flamencos en España); proyectos europeos (Policromia). Licenciado en Historia del Arte por la Universidad Autónoma de Madrid, diplomado en Restauración (CEROA), diplomado en Conservación y Restauración de Obras de Arte y Museología (UIA, Florencia). 


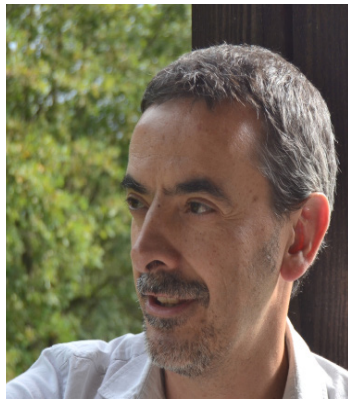

\section{Jesús Muñiz Petralanda}

jmpboreal@gmail.com

Licenciado en Historia del Arte (Universidad de Salamanca, 1988) y Diplomado en Especialización en Documentación y Gestión del Patrimonio Histórico (Universidad de Deusto, 1999). Entre 2002 y 2013 fue Conservador del Museo Diocesano de Arte Sacro de Bilbao, institución que editó su estudio sobre La Escultura Mueble del Gótico Tardío en Bizkaia. Se ha especializado en el estudio del arte mueble de la Baja Edad Media en el País Vasco, tema de la tesis doctoral que prepara actualmente.

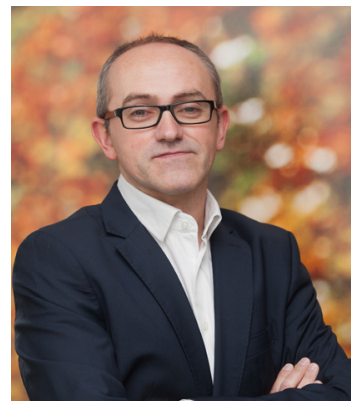

\section{Ignacio Migueliz Valcarlos}

Museo Universidad de Navarra

nmigueliz@unav.es

Licenciado en Geografía e Historia por la Universidad Autónoma de Madrid (1996) y doctor en Filosofía y Letras por la Universidad de Navarra (2004). Ha realizado el curso especializado de Museología de la Universidad San Pablo - CEU de Madrid (1997), y el curso de postgrado Gestión, Preservación y Difusión de Archivos Fotográficos en la Universidad Autónoma de Barcelona (2014). Ha obtenido la acreditación como Profesor Titular (2015) por la ANECA. Actualmente, y desde 2006, desarrolla su labor profesional como Conservador del Museo Universidad de Navarra, siendo Responsable de su Departamento Curatorial, y desde 2009 es profesor del departamento de Historia del Arte en el centro asociado de la UNED de Pamplona. Entre 2012 y 2015, fue profesor asociado del departamento de Historia, Historia del Arte y Geografía. Sus líneas de investigación son la fotografía, la platería, la joyería, la iconografía y la catalogación del patrimonio. En relación a estos temas ha comisariado y coordinado diferentes exposiciones y ha publicado libros, capítulos de libros y artículos en revistas especializadas.

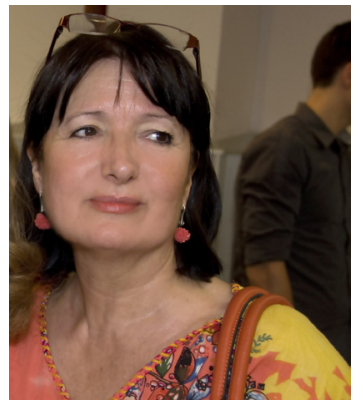

\section{María Pía Timón Tiemblo}

Instituto del Patrimonio Cultural de España

mpia.timon@mecd.es

Etnóloga de la Unidad de Patrimonio Cultural Inmaterial y Etnología del Instituto del Patrimonio Cultural de España (MECD). Ha obtenido diversos premios por trabajos de investigación relacionados con el Patrimonio Cultural: premio Matías Ramón Martínez (Asamblea de Extremadura), premio Juan de Goyeneche (Asamblea de Madrid) y premio Nacional de Investigación Marqués de Lozoya en Artes y Tradiciones populares. Ha publicado alrededor de 10 libros y unos100 artículos referidos a temas etnológicos. Imparte clases de Master sobre Patrimonio Etnológico en diferentes Universidades españolas y ha dirigido unos 20 cursos monográficos sobre Patrimonio Cultural Inmaterial. Es la representante española del Patrimonio Cultural Inmaterial en la JPI-Cultural Heritage del Consejo de Europa, coordinadora del Plan Nacional de Arquitectura Tradicional y del de Salvaguarda del Patrimonio Cultura Inmaterial. Ha comisariado recientemente la Exposición fotográfica de Inmaterial: Patrimonio y Memoria Colectiva que sigue estando itinerante por diferentes centros de España. 


\section{Itziar de Azua Brea}

Centro de Patrimonio Cultural del Gobierno Vasco

i-deazua@ej-gv.es

Licenciada en Historia por la universidad de Deusto. Procedente de la docencia en enseñanza secundaria (1982-1994), desde 1994 es técnico específico de Patrimonio Cultural en el Gobierno Vasco. Trabajó entre 1994 y 2000 en la Dirección de Patrimonio Cultural del Gobierno Vasco en el ámbito de Inventarios y Documentación del Patrimonio Cultural (Inventarios de Patrimonio histórico-arquitectónico, Patrimonio mueble de la Iglesia Católica, Inventario de Patrimonio Industrial e Inventarios específicos de puentes y Patrimonio pre-industrial). Tras un paréntesis- 2000-2009- en la Dirección de Universidades, se reincorporó al Centro de Patrimonio Cultural en 2009, desarrollando su actividad en relación a la información, catalogación y valoración del Patrimonio Cultural.

Artículo enviado el 18/10/2015

Artículo aceptado el 08/06/2016 